\title{
Lung Cancer Screening with Low-Dose CT: a Meta-Analysis
}

\author{
Richard M. Hoffman, MD, MPH ${ }^{1,2}$ (D), Rami P. Atallah, $M D^{3}$, Roger D. Struble, $M D^{7}$, and \\ Robert $G$. Badgett, $M D^{3}$
}

'University of lowa Carver College of Medicine, lowa City, IA, USA; ${ }^{2}$ Holden Comprehensive Cancer Center, University of lowa lowa City, IA, USA;

${ }^{3}$ The University of Kansas School of Medicine-Wichita, Wichita, KS, USA.

\begin{abstract}
BACKGROUND: Randomized controlled trials have evaluated the efficacy of low-dose CT (LDCT) lung cancer screening on lung cancer (LC) outcomes.

OBJECTIVE: Meta-analyze LDCT lung cancer screening trials.

METHODS: We identified studies by searching PubMed, Google Scholar, the Cochrane Registry, ClinicalTrials.gov, and reference lists from retrieved publications. We abstracted data on study design features, stage I LC diagnoses, LC and overall mortality, false positive results, harm from invasive diagnostic procedures, overdiagnosis, and significant incidental findings. We assessed study quality using the Cochrane risk-of-bias tool. We used randomeffects models to calculate relative risks and assessed effect modulators with subgroup analyses and metaregression.
\end{abstract}

RESULTS: We identified 9 studies that enrolled 96,559 subjects. The risk of bias across studies was judged to be low. Overall, LDCT screening significantly increased the detection of stage I LC, RR $=2.93$ (95\% CI, 2.16-3.98), $I^{2}=$ $19 \%$, and reduced LC mortality, RR $=0.84$ (95\% CI, 0.75$0.93), I^{2}=0 \%$. The number needed to screen to prevent an LC death was 265 . Women had a lower risk of LC death $(\mathrm{RR}=0.69,95 \% \mathrm{CI}, 0.40-1.21)$ than $\operatorname{men}(\mathrm{RR}=0.86,95 \%$ CI, 0.66-1.13), $p$ value for interaction $=0.11$. LDCT screening did not reduce overall mortality, $\mathrm{RR}=0.96$ (95\% CI, 0.91-1.01), $I^{2}=0 \%$. The pooled false positive rate was $8 \%$ (95\% CI, 4-18); subjects with false positive results had $<1$ in 1000 risk of major complications following invasive diagnostic procedures. The most valid estimates for overdiagnosis and significant incidental findings were $8.9 \%$ and $7.5 \%$, respectively.

DISCUSSION: LDCT screening significantly reduced LC mortality, though not overall mortality, with women appearing to benefit more than men. The estimated risks for false positive results, screening complications, overdiagnosis, and incidental findings were low. Long-term survival data were available only for North American and European studies limiting generalizability.

KEY WORDS: lung neoplasms; meta-analysis; mortality; low-dose computed tomography; lung cancer screening.

This study has not been presented previously.

Electronic supplementary material The online version of this article (https://doi.org/10.1007/s11606-020-05951-7) contains supplementary material, which is available to authorized users.

Received February 17, 2020

Accepted May 29, 2020

Published online June 24, 2020
J Gen Intern Med 35(10):3015-25

DOI: $10.1007 /$ s11606-020-05951-7

(C) Society of General Internal Medicine 2020

\section{INTRODUCTION}

Lung cancer is the most commonly diagnosed visceral cancer and the leading cause of cancer death in the world, with over 2 million new cases in 2018 and nearly 1.8 million deaths. ${ }^{1}$ Because over $90 \%$ of lung cancer deaths in the USA are attributed to tobacco use, tobacco control is the most impactful strategy for reducing the burden of lung cancer. ${ }^{2}$ However, combined pharmacotherapy and behavioral interventions benefit less than 1 in 10 smokers. ${ }^{3}$ In 2011, the National Lung Screening Trial (NLST) reported that screening high-risk smokers, ages 55 to 74, with low-dose CT (LDCT) significantly reduced lung cancer and overall mortality compared with screening with chest radiography. ${ }^{4}$ Although numerous other LDCT screening trials have been conducted worldwide, only the Nederlands-Leuvens Longkanker Screenings Onderzoek (NELSON) study, conducted in the Netherlands and Belgium, subsequently also demonstrated a statistically significant lung cancer mortality benefit for LDCT screening. ${ }^{5}$ The recently published NELSON trial had the 2nd largest sample size and the longest followup duration among all screening trials - and provided gender-specific outcome data. Recent meta-analyses, which did not include peer-reviewed NELSON results, showed that LDCT screening significantly reduced lung cancer mortality though not overall mortality. ${ }^{6-8}$ We conducted a metaanalysis to evaluate the association of LDCT lung cancer screening with early-stage cancer diagnoses, lung cancer mortality, overall mortality, and screening harms, including false positive results, complications from invasive procedures among subjects with false positive results, overdiagnosis, and significant incidental findings.

\section{METHODS}

We performed a review using the rapid review and living systematic methods supported by openMetaAnalysis. ${ }^{9}$ These reviews emphasize interpreting results more than searching by building on previous reviews. ${ }^{10}$ We began by creating a reconciliation table listing the included studies and conclusions 
from recent systematic reviews. ${ }^{11}$ This allowed us to readily identify the consistently included studies to be used in our meta-analysis as well as inconsistently included studies that at least two investigators carefully reviewed and discussed for inclusion. Identifying these studies also helped us design highsensitivity literature searches. Our data are maintained on the openMetaAnalysis site, enabling ongoing revisions by ourselves or others when new evidence becomes available.

We followed recommendations of PRISMA (Preferred Reporting Items for Systematic Reviews and Meta-Analyses) guidelines in conducting our meta-analysis, with the exception that the protocol was not registered at PROSPERO because it does not support living systematic reviews. ${ }^{12}$ The PRISMA checklist is in the supplemental index.

\section{Study Eligibility Criteria and Selection}

We included randomized controlled trials of computed tomography $(\mathrm{CT})$ that reported lung cancer and/or overall mortality data. Studies were selected by the consensus of the four authors.

\section{Information Sources}

We first identified trials by tabulating all trials included in the most recent systematic reviews (published since 2016) ${ }^{6-8,13}$, ${ }^{14}$ as recommended by Riaz. ${ }^{15}$

\section{Search Strategy}

We executed three search strategies in PubMed. We first performed a Boolean PubMed search from January 2017, the date of the most recent comprehensive literature review, ${ }^{8}$ until April 2020 using search terms chosen to identify all studies in the reconciliation of studies table. We then executed a vector search for the same time period using PubMed's "Find related data" option to search conceptually similar studies to those included in the reconciliation of studies table. The vector was simultaneously seeded with all studies in the reconciliation table. An experienced librarian developed an Ovid MEDLINE search using terms for lung neoplasms, screening and early detection of cancer, computed tomography, and randomized trials (supplement table 2). We limited the Ovid search to English language studies published from January 2011 until April 2020 to ensure that we retrieved all studies published after the NLST.

We also searched Google Scholar and Web of Science with a strategy which retrieved articles published since January 2017 that cited the NLST (the most highly cited study) and contained the terms "randomized" and "mortality." We additionally searched ClinicalTrials.gov and the Cochrane Central Register of Controlled Trials. Finally, we created a monthly email alert to be notified if new articles are published in PubMed that contain our Boolean search terms or specific text words. Two reviewers screened titles and abstracts for review eligibility.

\section{Study Selection Process}

We reviewed abstracts to identify publications from randomized trials and then retrieved relevant full-text publications. We retrieved multiple publications from a given trial in order to extract comprehensive data on study design, baseline characteristics, and outcomes. At least 2 reviewers were involved in all decisions regarding retrieval of full-text manuscripts.

\section{Data Collection Process}

We collected the following clinical data from randomized controlled trials of LDCT lung cancer screening: country, year first subject enrolled, number of clinical sites, mean (median) age, percent $<65$ years, percent male, mean (median) pack-years smoking, proportion currently smoking, screening and control interventions, rounds of screening, screening intervals, follow-up duration, incident cancers and proportion that were early stage, lung cancer deaths, and overall mortality. When provided, we also abstracted data by gender. We abstracted data on potential harms of screening, including false positive tests, complications from invasive procedures performed in subjects without cancer, overdiagnosis, and significant incidental findings. We entered clinical data from studies into online spreadsheets at openMetaAnalysis. ${ }^{9}$

\section{Risk of Bias in Individual Studies}

Four authors independently assessed the risk of bias by using the Cochrane Risk of Bias Tool version $1 .^{16}$ These assessments were then reviewed by conference with all authors to resolve any disagreement. The risk of bias was reported as low, high, or unclear based on the items in the Cochrane tool. We considered individual studies at high risk of bias if the study had high risk of bias for one or more key domains of the Cochrane tool. We considered individual studies at unclear risk of bias if the study had an unclear risk of bias for one or more key domains and no high-risk domains.

\section{Summary Measures}

Our primary outcomes were lung cancer-specific mortality and overall mortality. Our secondary outcomes were diagnosis of early-stage (stage I) lung cancer and harms from screening. We reported relative risks with $95 \%$ confidence intervals (CI).

\section{Synthesis of Results}

We used a random-effects model with the Hartung-Knapp estimator. ${ }^{17}$ We performed all statistical analyses online at openCPU ${ }^{18}$ with the meta package of the $\mathrm{R}$ programming language. ${ }^{19}$ We measured heterogeneity of results with the $I^{2}$ statistic. $^{20,21}$

We used subgroup and meta-regression analyses to characterize potential modulators of screening effectiveness. We 
conducted subgroup analyses based on the control intervention (chest X-ray or usual care), by gender, and risk of bias. We used meta-regression to examine modulators of the screening effect on lung cancer and overall mortality, including age (mean/median and percent $<65$ years), proportion of male subjects, pack-year smoking history, percent current smokers, number of screening rounds, screening intervals, proportion of cancers diagnosed at stage I, and duration of follow-up. If meta-regression identified a statistically significant modulator, we would identify the cut point (threshold) of the modulator that was associated with a statistically significant reduction in mortality.

\section{Risk of Bias Across Studies}

We judged the overall risk of bias across the collected studies based on the criteria developed by the Cochrane Risk of Bias Tool. ${ }^{16}$ These criteria consider the risk of bias low if less than $25 \%$ of participants were from studies with low methodological quality. The risk of bias is considered serious if more than $25 \%$ but less than $50 \%$ of participants were from studies with low methodological quality and is considered very serious if more than $50 \%$ of participants were from studies with low methodological quality. We assessed publication bias using funnel plot asymmetry and Egger's and Rucker's tests. ${ }^{20,} 22,23$

We summarized conclusions using the Grade Working Group's Evidence Profile and Summary of Findings Table. ${ }^{24}$ We adjusted ratings based on the criteria developed by the Cochrane Back Group ${ }^{25}$ and described online at openMetaAnalysis. ${ }^{9}$

\section{Data Availability}

The literature search, datasets generated and analyzed during the current study, all plots, and the tables reconciling our conclusions and the trials included with previous metaanalyses are available online (https://openmetaanalysis. github.io/lung-cancer-screening).

\section{RESULTS}

\section{Study Selection}

We identified 9 randomized controlled trials from reference lists of meta-analyses (Fig. 1). ${ }^{4}, 5,26-32 \mathrm{We}$ also identified 3663 citations through extensive literature searching. However, we did not find any additional eligible randomized controlled trials beyond those identified from the meta-analyses.

\section{Study Characteristics}

Studies are described in Table 1. The 9 studies enrolled a total of 96,559 subjects. The mean and median age was around 60 ,
$64.1 \%$ were male, $51.7 \%$ were current smokers, and the mean and median pack-years of smoking was usually about 40 or more. We found 7 comparisons of LDCT screening vs. usual care (one study performed a baseline chest X-ray and sputum cytology for all subjects) ${ }^{35}$ and 2 comparisons of LDCT with chest X-rays. ${ }^{4,24}$ Aside from the pilot Lung Screening Study ${ }^{26}$ and the AME Thoracic Surgery Collaborative Group (AME) trial reporting only baseline results, ${ }^{32}$ subjects in the LDCT arms underwent 3 to 5 rounds of screening. The latter studies generally had a mean or median follow-up duration of at least 8 years. In the 8 studies reporting cancer incidence data, 1910 lung cancers were found in the LDCT arms and 1578 in the control arms. Overall, $48.5 \%$ of cancers in the LDCT arms were detected at stage I compared with $24.3 \%$ in the control arms. In the LDCT arms, there were 890 lung cancer deaths and 3755 overall deaths. In the control arms, there were 1062 lung cancer deaths and 3912 overall deaths.

We evaluated only the 3446 subjects from the Italian Multicentric Italian Lung Detection (MILD) study that were concurrently enrolled and randomly assigned to the screening arms of LDCT or usual care. ${ }^{29}$ Although included by the authors in their reports, we excluded the 653 subjects who were randomized to either annual or biennial screening but not to a control group during the first phase of the study. We also included 92 subjects (46 in each arm) in the Italian study Detection and Screening of Early Lung Cancer by Novel Imaging Technology and Molecular Essays (DANTE) that investigators had excluded for being ineligible. ${ }^{27} \mathrm{We}$ did so because these subjects had consented to participate in the study, underwent clinical assessment, and were randomly allocated to study arms. The DANTE authors provided data on lung cancer and overall mortality, so we included these subjects in an intention-to-screen analysis. Although the NELSON report focused on outcomes for male patients, our analyses incorporated the data for female patients published in the supplemental index. ${ }^{5}$

We did not include the most recently published NLST outcome data, which provided a median 12.3 years of mortality follow-up..$^{60}$ These data, based on deaths occurring up to 10 years after the final scheduled screen, showed attenuation of mortality benefits. However, participants were only passively followed through state tumor registry and National Death Index linkages, and cause of death was not adjudicated - raising concerns about bias. Furthermore, as recognized by the investigators, the longerterm follow-up diluted the screening effect making results less comparable to the other studies.

\section{Risk of Bias Within Studies}

The risk of bias table is in the supplemental index. We assessed the MILD, DANTE, and AME studies as being at high risk of bias, the NLST and NELSON trials as being at low risk of bias, and the remaining studies as being at unclear risk of bias. The MILD study failed to provide baseline comparisons of contemporaneously enrolled screening and control 


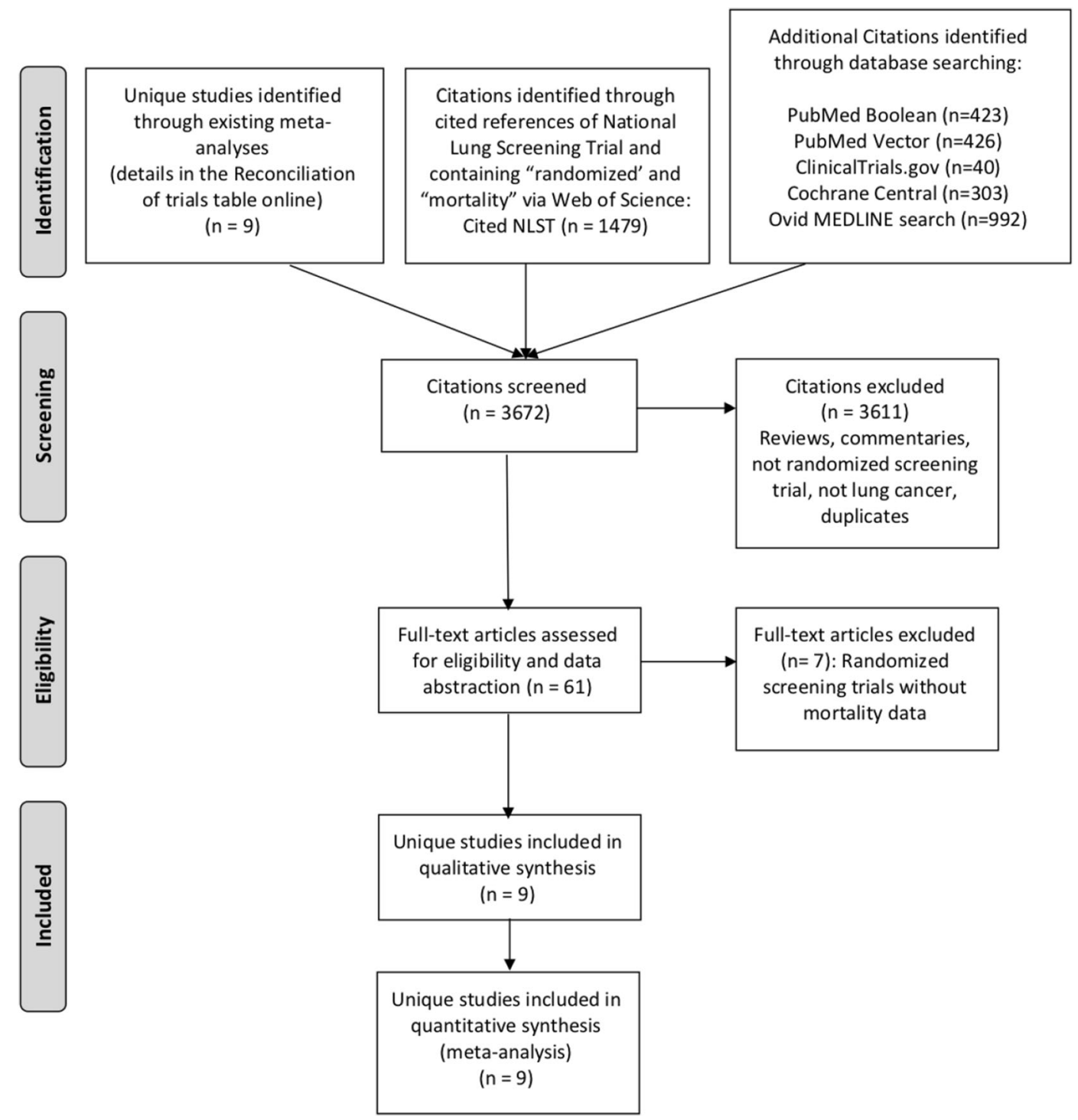

Fig. 1 PRISMA flow diagram.

subjects. The DANTE investigators did not use an intentionto-screen analysis and enrolled $5.5 \%$ more subjects in the screening arm than in the control arm. The AME study did not describe randomization procedures and had nearly $12 \%$ more subjects in the screening arm than in the control arm.

\section{Synthesis of Results}

When we pooled results across the 8 trials reporting data, we found that lung cancer screening with LDCT was associated with a significantly increased likelihood of detecting a stage I lung cancer, $\mathrm{RR}=2.73$ (95\% CI, 1.90-3.91), but heterogeneity was high: $I^{2}=79 \%$ (Fig. 2). However, when we restricted the analysis to the 7 studies without a screened control group, the relative risk for diagnosing stage I cancer was 2.93 (95\% CI, 2.16-3.98), with much lower heterogeneity: $I^{2}=19 \%$.

Lung cancer screening with LDCT significantly reduced the risk of dying from lung cancer, with a relative risk of 0.84 (95\% CI, 0.75-0.93) (Fig. 3). Heterogeneity was very low, with an $I^{2}=0 \%$. Overall, the number needed to screen to prevent one lung cancer death, based on 3 to 5 rounds of screening with up to 10 years of follow-up, was 265 .

We evaluated the effects of screening on lung cancer mortality stratified by gender in the 3 studies reporting these data (Fig. 4). Screening decreased lung cancer mortality among women, but the relative risk of 0.69 (95\% CI, 0.40-1.21) was not significant. Results were also not significant for men, with a relative risk of 0.86 (95\% CI, 0.66-1.13). A test for interaction was not significant, $p=0.11$. We did not find any subgroup effects when stratifying studies by control group or risk of bias. We conducted meta-regression analyses evaluating associations between patient characteristics and study factors with lung cancer mortality. We found no significant effects by median/mean age, percent $<65$ years, proportion of male subjects, pack-year history, percent current smokers, number and frequency of screening rounds, proportion of cancers found at stage I, and duration of follow-up (data 
Table 1 Study Characteristics

\begin{tabular}{|c|c|c|c|c|c|c|}
\hline $\begin{array}{l}\text { Study name, country, enrollment } \\
\text { years, clinical sites, eligibility } \\
\text { criteria, number of randomized } \\
\text { subjects }\end{array}$ & $\begin{array}{l}\text { Age mean } \\
\text { (SD); median } \\
\text { (IQR); range } \\
(\%) \\
\%<65\end{array}$ & $\begin{array}{l}\text { Male } \\
(\%)\end{array}$ & $\begin{array}{l}\text { Pack-years mean } \\
\text { (SD); median } \\
\text { (IQR); range }(\%)\end{array}$ & $\begin{array}{l}\text { Current } \\
\text { smokers } \\
(\%)\end{array}$ & $\begin{array}{l}\text { LDCT screening and } \\
\text { comparator }(N)\end{array}$ & $\begin{array}{l}\text { Follow-up } \\
\text { (years) mean } \\
\text { (SD); median } \\
\text { (IQR) }\end{array}$ \\
\hline${ }_{34}$ ung Screening Study (LSS) 26,33 , & $\begin{array}{l}55-59(38.5) \\
60-64(31.2)\end{array}$ & 58.6 & $\begin{array}{l}<40(17.8) \\
40-55(33.5)\end{array}$ & 57.5 & $\begin{array}{l}\text { LDCT, } 2 \text { annual rounds } \\
(N=1660)\end{array}$ & 5.2 (IQR NR) \\
\hline US, 2000-2001 & $65-69(21.2)$ & & $55-75(22.9)$ & & Control, CXR, 2 annual & \\
\hline 6 sites & $70-74(11.8)$ & & $75+(24.8)$ & & rounds $(N=1658)$ & \\
\hline Ages 55-74 & $69.7 \%<65$ & & & & & \\
\hline
\end{tabular}

Smoking history:

$\geq 30$ pack-years

Current or quit $\leq 10$ years

$N=3318$

DANTE $^{27,35-37}$

Italy, 2001-2006

3 sites

Men ages 60-74

Smoking history: $\geq 20$ pack-years

Current or quit $\leq 10$ years ago

$N=2532$

$\begin{aligned} & 64.0 \text { (IQR 5) } \\ & \%<65 \text { NR }\end{aligned} \quad 100.0 \quad 47.3($ IQR 2.4)

56.9

$\%<65 \mathrm{NR}$

cytology, 5 annual

National Lung Screening Trial

$(\mathrm{NLST})^{4}$

US, 2002-2004

$61.4(\mathrm{SD} 5.0)$

$59.0 \quad 48$ (SD NR)

48.2

$73.4 \%<65$

33 sites

Ages 55-74

Smoking history: $\geq 30$ pack-years

Current or quit $\leq 15$ years

$N=53,452$

Nederlands Leuvens Longkanker

Screeningsonderzoek (NELSON) ${ }^{5}$,

Belgium/Netherlands, 2004-2006

4 sites

Smoking history: 25 years $\geq 3 / 4$

pack, 30 years $>1 / 2$ pack

Quit $\leq 10$ years Exclude lung

cancer $<5$ years and/or on treat-

ment

$N=15,792$

Danish Lung Cancer Screening

Trial (DLCST) ${ }^{28,50-52}$

Denmark, 2004-2006

1 site

Ages 50-70

Smoking history: $\geq 20$ pack-years

Current or quit within 10 years

(and at age $>50$ )

$N=4104$

Italian Lung study (ITALUNG)

Italy, 2004-2006

3 sites

Ages 55-69

Smoking history: $\geq 20$ pack-years

Current or quit $<10$ years

$N=3206$

Multicentric Italian Lung

Detection (MILD) ${ }^{29,55-57}$

Italy, 2005-2011

1 site

Ages 49 to 75

Tobacco use: $\geq 20$ pack-years

Current or quit $<10$ years

$N=3446$

German Lung Cancer Screening

Intervention Trial (LUSI) ${ }^{31,58}$, 59

Germany, 2007-2011

1 region

Ages 50-69

Smoking history: 25 years $\geq 3 / 4$

pack, 30 years $>1 / 2$ pack
58 (IQR 8)

$82.7 \%<65$

83.6 38 (IQR 19.8)

55.5

Screening

57.9 (SD 4.8)

Control

47.8 (SD 4.8)

$91.0 \%<65$

60.7 (SD NR)

$70.6 \%<65$

$64.7 \quad 41.6$ (SD NR)

64.5

Screening

36.4 (SD 13.4)

Control

35.9 (SD 13.4)

76.1

Control

Intervention

58 (IQR NR)

$86.3 \%<65$

55 (IQR NR)

$72.1 \%<65$

64.5 Control

38 (IQR NR)

Intervention

39 (IQR NR)

$64.7 \quad \mathrm{NR}$

61.9
57 (IQR NR)
LCDT, 5 annual rounds $(N=2029)$

Control, 5 annual

surveys $(N=2023)$ rounds of LDCT $(N=$

1300)

Control, baseline

radiograph and sputum

cytology, 5 annual

clinical review $(N=$

1232)

LDCT, 4 rounds:

baseline, 1 year, 3

years, 5.5 years $(N=$

7900)

Control, no screening

$(N=7892)$

Baseline, year 1, year 3, year 5.5

LDCT, 5 annual rounds $(N=2052)$

Control, 5 annual health interviews $(N=2052)$

LDCT, 4 annual rounds $(N=1613)$

Control, usual care $(N=$ 1593)

8.35 (IQR NR)

6.5 (IQR NR)

10 (minimum)

9.8 (IQR NR)

9.3 (IQR, 8.89.9)

$\begin{array}{ll}\text { LDCT, } 3 \text { or } 5 \text { rounds }(N & 9(93.5) \\ =1723 ; 864 \text { annual, } & 10(71.0)\end{array}$

859 biennial)

Usual care $(N=1723)$

8.89 (IQR NR) 
Table 1. (continued)

\begin{tabular}{|c|c|c|c|c|c|c|}
\hline $\begin{array}{l}\text { Study name, country, enrollment } \\
\text { years, clinical sites, eligibility } \\
\text { criteria, number of randomized } \\
\text { subjects }\end{array}$ & $\begin{array}{l}\text { Age mean } \\
\text { (SD); median } \\
\text { (IQR); range } \\
(\%) \\
\%<65\end{array}$ & $\begin{array}{l}\text { Male } \\
(\%)\end{array}$ & $\begin{array}{l}\text { Pack-years mean } \\
\text { (SD); median } \\
\text { (IQR); range }(\%)\end{array}$ & $\begin{array}{l}\text { Current } \\
\text { smokers } \\
(\%)\end{array}$ & $\begin{array}{l}\text { LDCT screening and } \\
\text { comparator }(N)\end{array}$ & $\begin{array}{l}\text { Follow-up } \\
\text { (years) mean } \\
\text { (SD); median } \\
\text { (IQR) }\end{array}$ \\
\hline $\begin{array}{l}\text { Current or quit } \leq 10 \text { years } \\
N=4052 \\
\text { AME Thoracic Surgery } \\
\text { Collaborative Group } \\
\text { China, 2013-2014 } \\
1 \text { site } \\
\text { Ages } 45-70 \\
\text { Smoking history: } \geq 20 \text { PY } \\
\text { Current or quit }<15 \text { years } \\
\text { Other environmental, personal, or } \\
\text { family risk factors } \\
N=6679\end{array}$ & $\begin{array}{l}59.8(\mathrm{SD} 5.8) \\
\%<65 \mathrm{NR}\end{array}$ & 46.8 & $\begin{array}{l}\geq 30(8.2 \%) \\
\geq 20-30(6.8 \%) \\
<20(12.9 \%) \\
\text { Other risk factors } \\
\text { Cooking oil } \\
\text { exposure }(61.2 \%) \\
\text { Carcinogenic } \\
\text { occupational } \\
\text { exposures }(9.3 \%)\end{array}$ & 21.5 & $\begin{array}{l}\text { LDCT, } 2 \text { biennial } \\
\text { rounds }(N=3512) \\
\text { Control, standard care } \\
(N=3145)\end{array}$ & $\begin{array}{l}2(\mathrm{SD}, \mathrm{IQR} \\
\mathrm{NR})\end{array}$ \\
\hline
\end{tabular}

LDCT, low-dose computed tomography; NR, not reported; IQR, interquartile range; $S D$, standard deviation

available at https://openmetaanalysis.github.io/lung-cancerscreening).

When pooling results from across the eight studies reporting data, we found that lung cancer screening did not significantly reduce the risk of overall mortality, the relative risk was 0.96 (95\% CI, 0.91-1.01) (Fig. 5). We found no heterogeneity in study results, $I^{2}=0 \%$.

Screening harms are detailed in the supplemental index. Eight studies reported diagnostic accuracy data, the pooled false positive rate was $8 \%(95 \%$ CI, $4-15), I^{2}=100 \%$. Studies inconsistently reported data on complications from invasive diagnostic procedures, particularly among those without lung cancer, but the risks were low. Only the NLST provided detailed data on procedures and associated complications (which were further classified by severity). Overall, 17 in 1000 subjects with a false positive LDCT underwent an invasive diagnostic procedure and 0.4 in 1000 suffered a major complication. Six studies compared LDCT with usual care and followed patients beyond the end of the screening period. Overall, there were 515 screen-detected cancers with 171 more cancers in the LDCT arms than in the control arms, suggesting an overdiagnosis rate of $33 \%$. The NLST reported

Diagnosis of stage I lung cancer

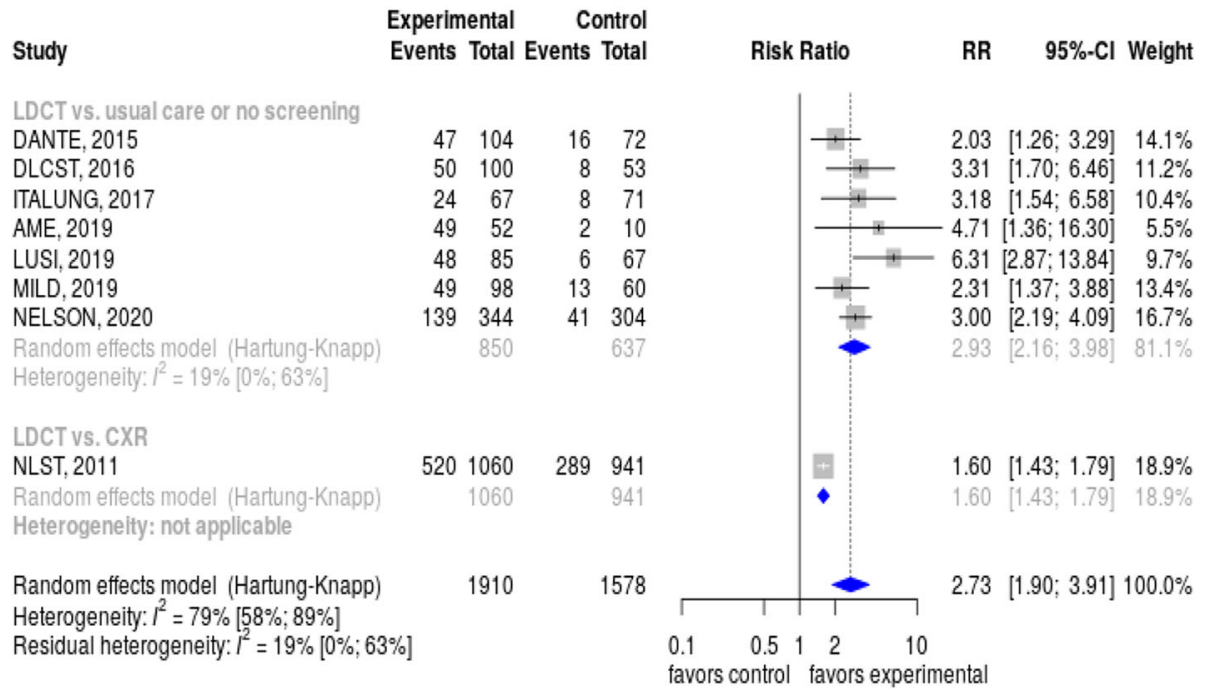


Lung cancer mortality

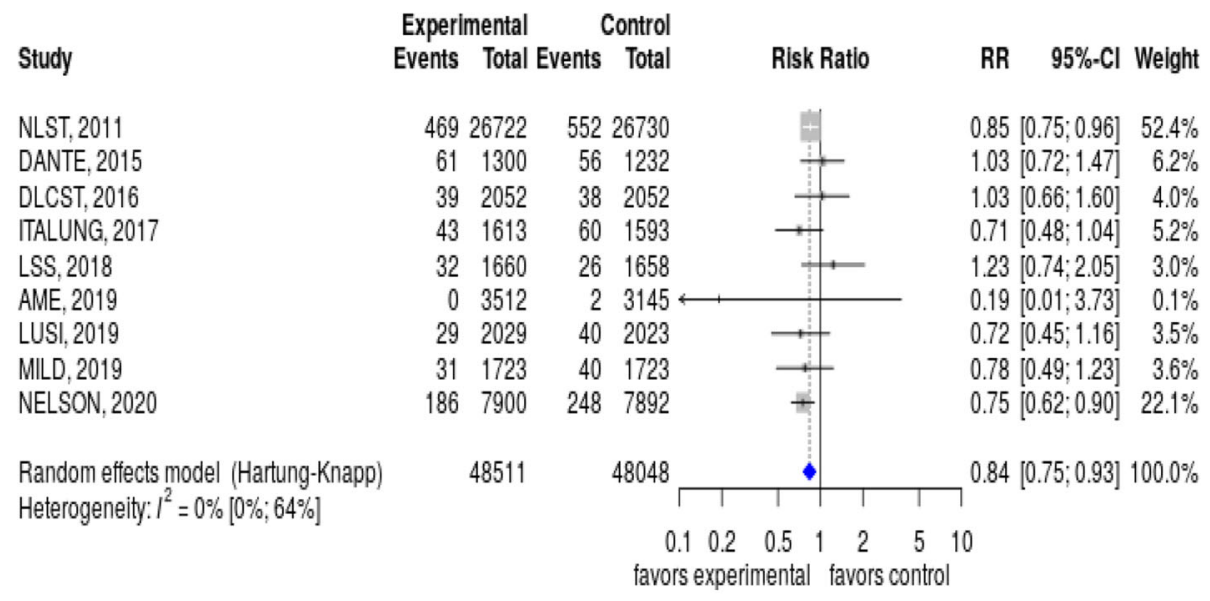

Test for funnel plot asymmetry: too few studies to test

Fig. 3 Forest plot: Lung cancer mortality.

that $7.5 \%$ of LDCT participants had significant incidental findings, most commonly emphysema and coronary artery calcification. ${ }^{4}$ No other studies reported data on incidental findings.

The GRADE evidence profile, summarizing quality assessments and effects, is in the supplemental index. We had high certainty about the effects of LDCT screening on the detection of stage I cancer, lung cancer mortality, and overall mortality.

\section{Risk of Bias Across Studies}

We judged the risk of bias across studies to be low using the Cochrane framework. Although the majority of individual studies were rated as unclear risk of bias, more than threefourths of the subjects were enrolled in the two studies assessed to be at low risk of bias. ${ }^{4,5} \mathrm{We}$ could not assess publication bias because we found too few studies $(<10)$ to perform analyses. $^{61}$

\section{DISCUSSION}

We evaluated 9 randomized LDCT screening trials enrolling over 96,000 subjects most of whom were followed for at least 5 years. LDCT screening substantially increased the likelihood of detecting stage I cancer and reduced the risk of lung cancer mortality by $16 \%$. Heterogeneity was very low, and the quality of the evidence was deemed to be moderate to high quality. When we evaluated gender-specific effects, we found that LDCT screening was associated with a non-significantly lower risk of lung cancer mortality for women than for men. Meta-regressions found no subject characteristics or study design features that modified lung cancer mortality outcome results. LDCT screening did not reduce overall mortality.
Our results are similar to previous meta-analyses, though we included the full peer-reviewed NELSON trial results to provide more accurate outcome estimates and to better evaluate gender differences. Evidence is convincing that LDCT screening reduces lung cancer mortality. LDCT screening is effective because it leads to a nearly threefold higher likelihood of diagnosing early-stage cancer compared with usual care. This stage shift is crucial, because about $60 \%$ of patients are currently diagnosed with distant-stage disease where the 5year survival is only $5 \% .^{62}$ In contrast, only $16 \%$ of patients are diagnosed with early-stage cancer where the 5-year survival is $57 \%$. Needing to screen 265 high-risk smokers to prevent one lung cancer death compares favorably with other cancer screening programs. ${ }^{63-65}$

Increasing the proportion of cancers detected at an early stage can also be associated with overdiagnosing - and overtreating - indolent cancers. Although 33\% of cancers found by LDCT screening might be considered overdiagnosed, this estimate is unreliable because most studies lacked sufficient follow-up time. Modeling analyses suggested that the lead time for CT screening can be as long as 12 years. ${ }^{66}$ The NELSON investigators initially estimated an overdiagnosis rate of $19.7 \%$ through 10 years of followup; however, extending follow-up to 11 years reduced the rate to only $8.9 \% .^{5}$ Investigators concluded that this prolonged follow-up duration was necessary to accurately estimate overdiagnosis. We excluded NLST when estimating overdiagnosis because both arms were screened. However, trends in NLST results were consistent with NELSON findings; the overdiagnosis rate dropped from $18.5 \%$ at 6.5 years of follow-up to $3.1 \%$ at 11 years of followup. $^{38,60}$ 
Lung cancer mortality by gender

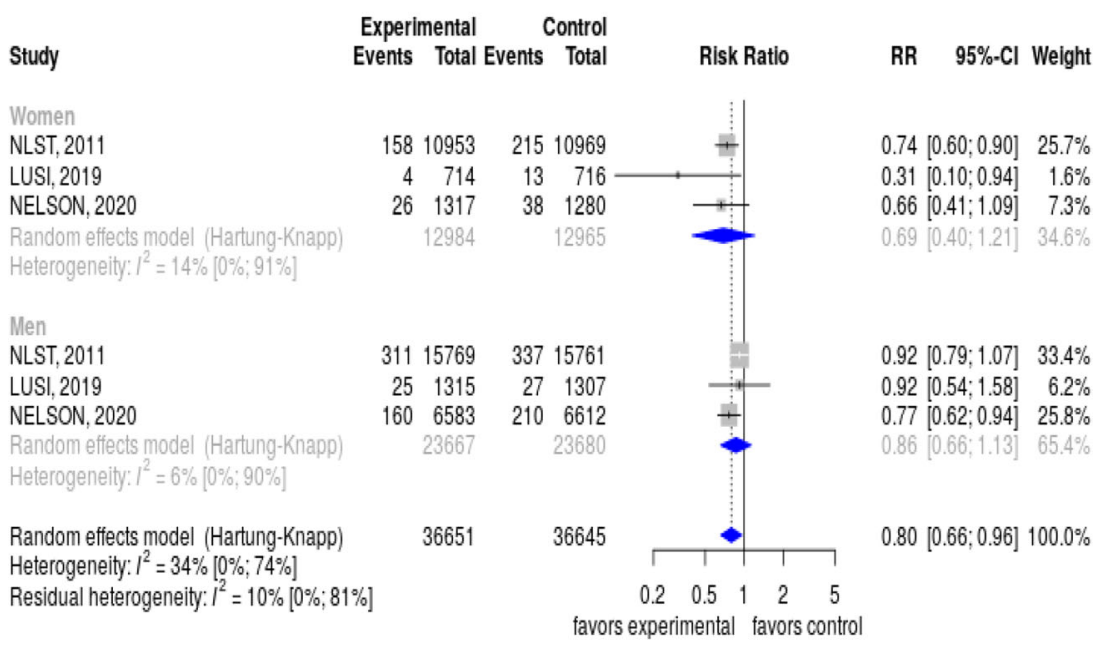

Test for differences among subgroups: $p=0.115$

Test for funnel plot asymmetry: too few studies to test

Fig. 4 Forest plot: Lung cancer mortality by gender.

False positive results are a potential harm from LDCT screening because they can lead to unnecessary diagnostic testing. The estimated false positive rate was $8 \%$, but heterogeneity was extremely high because studies used varying criteria for categorizing abnormal tests (supplement table 4). The NLST, which primarily defined positive studies using a nodule diameter $\geq 4$ $\mathrm{mm}$, had a false positive rate of $23.3 \% .^{4}$ However, the LungRADS classification system, which increases the size threshold for identifying suspicious scans, has since become the standard for interpreting LDCT images. ${ }^{67}$ Post-hoc analyses suggested that applying Lung-RADS to NLST images would substantially reduce the false positive rates for both baseline (52\% decrease) and follow-up (76\% decrease) testing, though at the expense of reducing sensitivity. ${ }^{68}$ The NELSON trial, which had a false positive rate of only $1.2 \%$, used volumetric criteria which are not part of Lung-RADS. ${ }^{5}$ The NLST provided the most detailed data on complications following invasive diagnostic procedures in subjects without cancer, the risk was very low though patients were being managed in academic medical centers. The NLST, the only study to provide data, found a $7.5 \%$ chance of having a significant

\section{All cause mortality}

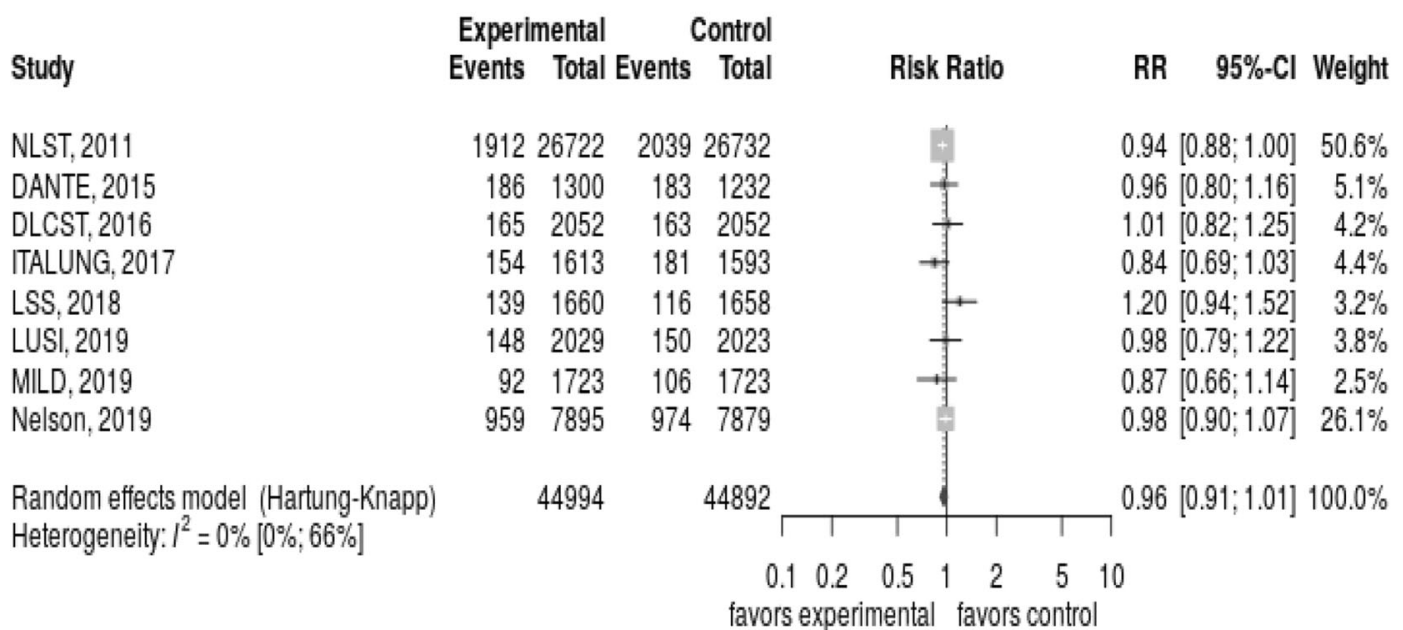

Test for funnel plot asymmetrv: too few studies to test

Fig. 5 Forest plot: Overall mortality. 
incidental finding with LDCT imaging. However, the clinical significance of these findings is uncertain.

When we pooled results across all studies, screening was associated with a non-significant decreased risk for overall mortality. The NLST was the only trial showing lower overall mortality with LDCT screening. The authors attributed this finding to the high proportion of excess deaths from lung cancer in the radiography arm. When lung cancer deaths were excluded, the overall mortality difference was not significant. Generally, trials are unlikely to demonstrate that screening programs, which usually target average-risk subjects, decrease overall mortality because even the most common cancers account for only a small proportion of deaths. While lung cancer accounts for more deaths in the high-risk populations selected for screening, a modeling study suggested that more than 80,000 high-risk subjects would need to be randomized to a screening or a control arm and followed for at least 11 to 13 years in order to demonstrate a significant reduction in allcause mortality. ${ }^{69}$ While we had pooled data for nearly 100,000 subjects, follow-up durations were usually less than 10 years. A further challenge in demonstrating that lung cancer screening reduces overall mortality is that the eligible population of older heavy smokers is also at high risk for competing mortality from tobacco-related cardiovascular, pulmonary, and oncologic diseases.

By including results from NELSON, which enrolled 2594 women, we were able to conduct a more robust meta-analysis of the three studies that stratified outcome data by gender., ${ }^{5,31}$, ${ }^{70} \mathrm{We}$ found that women benefitted from screening substantially more than men, $31 \%$ relative risk reduction in lung cancer mortality compared with $14 \%$. Neither risk reductions were statistically significant and the $p$ value for interaction was 0.11 . These studies enrolled nearly twice as many men as women, so analyses for women were likely underpowered. Unfortunately, we did not have access to patient-level data for the 5 other studies that also enrolled men and women. The mortality benefit for women in NLST was attributed to better outcomes following the diagnosis of small cell and squamous cell lung cancers. ${ }^{70}$ Given that screening is not considered effective for detecting these lung cancers (and risk reductions were similar for men and women for adenocarcinoma), investigators questioned whether the findings were due to chance. However, in the much smaller LUSI trial, which found a significant $69 \%$ risk reduction for women, no small cell cancers were diagnosed in women. ${ }^{31}$ The NELSON trial, which found a non-significant $34 \%$ risk reduction for women, did not report histology. ${ }^{5}$ Further research is needed to evaluate the observed gender differences.

The negative meta-regression analyses looking at the associations between screening protocols and patient characteristics with lung cancer mortality are underpowered given the limited number of studies. However, the optimal number and frequency of LDCT screening rounds are uncertain. ${ }^{71}$ The UK Lung Cancer Screening Trial will evaluate the benefit of a single screening LDCT. ${ }^{72}$ For the 2 studies demonstrating efficacy for LDCT screening, the NLST had 3 rounds of annual screening while the NELSON trial spaced 4 rounds of screening over 5.5 years. Less frequent screening intervals, particularly following a negative baseline scan, could make screening more cost effective and, along with using LungRADS to improve the specificity of LDCT, reduce radiation exposure from screening and diagnostic testing. ${ }^{68,73,74} \mathrm{We}$ did not find that the patient characteristics of age, pack-years of smoking, or smoking status (typically used to determine screening eligibility) were associated with the lung cancer mortality benefits seen with screening. However, the variation of these characteristics across studies was small, particularly for age and pack-years (Table 1). Tammemagi and colleagues have shown that using comprehensive risk models, which include additional socio-demographic characteristics, clinical features, and family history, to select patients for screening may be more cost effective than using the study trial inclusion criteria that have been adopted for screening guidelines. ${ }^{75}$ The U.S. Preventive Services Task Force's recommendation for lung cancer screening is being revised and will address eligibility criteria, including age range and calculated cancer risk, as well as alternatives to annual screening. ${ }^{71}$

Our study had several additional limitations. Long-term mortality data were available only for studies conducted in Europe and North American, which may limit the generalizability of results based on screening just older, high-risk current or former smokers. Lung cancer incidence and mortality rates vary around the world, particularly in emerging economies and developing countries, related to differences in genetics, tobacco use, environmental exposures, and access to care. ${ }^{76}$ The Chinese AME trial enrolled substantial proportions of subjects whose lung cancer risk was defined as exposure to second-hand smoke, cooking oil fumes, or occupational carcinogens. ${ }^{32}$ Additionally, the observed efficacy of LDCT screening as conducted in randomized clinical trial settings may not translate into community practice. ${ }^{77}$

\section{CONCLUSION}

Our meta-analysis, utilizing the most recently published randomized controlled trial data, demonstrated that LDCT screening is associated with a significant reduction of lung cancer mortality though not overall mortality. Women appeared more likely to benefit from screening than men, but data were inconclusive. The estimated risks for false positive results, screening complications, overdiagnosis, and incidental findings were low.

Acknowledgments: We appreciate the literature search assistance provided by Heather Healy, Clinical Education Librarian, University of Iowa Hardin Library for the Health Sciences.

Corresponding Author: Richard M. Hoffman, MD, MPH; Holden Comprehensive Cancer Center, University of Iowa Iowa City, IA, USA (e-mail: richard-m-hoffman@uiowa.edu). 
Funding Information Dr. Hoffman's cancer-related work is supported by Cancer Center Support Grant PO3O CAO86862

\section{Compliance with ethical standards:}

Conflict of Interest: The authors declare that they do not have a conflict of interest.

\section{REFERENCES}

1. Bray F, Ferlay J, Soerjomataram I, Siegel RL, Torre LA, Jemal A. Global cancer statistics 2018: GLOBOCAN estimates of incidence and mortality worldwide for 36 cancers in 185 countries. CA Cancer $\mathrm{J}$ Clin. 2018;68:394-424.

2. US Department of Health and Human Services. The health consequences of smoking: 50 years of progress. A report of the Surgeon General. Atlanta, GA: U.S. Department of Health and Human Services, Centers for Disease Control and Prevention, Natonal Center for Chronic Disease Prevention and Health Promotion, Office on Smoking and Health; 2014.

3. Stead LF, Koilpillai P, Fanshawe TR, Lancaster T. Combined pharmacotherapy and behavioural interventions for smoking cessation. Cochrane Database Syst Rev. 2016;3:CD008286.

4. National Lung Screening Trial Research Team, Aberle DR, Adams AM, et al. Reduced lung-cancer mortality with low-dose computed tomographic screening. N Engl J Med. 2011;365:395-409.

5. de Koning HJ, van der Aalst CM, de Jong PA, et al. Reduced LungCancer Mortality with Volume CT Screening in a Randomized Trial. N Engl J Med. 2020;382:503-13.

6. Huang KL, Wang SY, Lu WC, Chang YH, Su J, Lu YT. Effects of low-dose computed tomography on lung cancer screening: a systematic review, meta-analysis, and trial sequential analysis. BMC Pulm Med. 2019; 19: 126 .

7. Rota M, Pizzato M, La Vecchia C, Boffetta P. Efficacy of lung cancer screening appears to increase with prolonged intervention: results from the MILD trial and a meta-analysis. Ann Oncol. 2019;30:1040-3.

8. Snowsill T, Yang H, Griffin E, et al. Low-dose computed tomography for lung cancer screening in high-risk populations: a systematic review and economic evaluation. Health Technol Assess. 2018;22:1-276.

9. openMetaAnalysis. Methods. (Accessed February 2, 2020, at http:// openmetaanalysis.github.io/methods.html)

10. Patnode CD, Eder ML, Walsh ES, Viswanathan M, Lin JS. The Use of Rapid Review Methods for the U.S. Preventive Services Task Force. Am J Prev Med. 2018;54:S19-S25.

11. Riaz IB, Khan MS, Riaz H, Goldberg RJ. Disorganized Systematic Reviews and Meta-analyses: Time to Systematize the Conduct and Publication of These Study Overviews? Am J Med. 2016;129:339 e11-8.

12. Liberati A, Altman DG, Tetzlaff $\mathbf{J}$, et al. The PRISMA statement for reporting systematic reviews and meta-analyses of studies that evaluate health care interventions: explanation and elaboration. Ann Intern Med. 2009; 151:W65-94.

13. Coureau G, Salmi LR, Etard C, Sancho-Garnier H, Sauvaget C, Mathoulin-Pelissier S. Low-dose computed tomography screening for lung cancer in populations highly exposed to tobacco: A systematic methodological appraisal of published randomised controlled trials. Eur $\mathrm{J}$ Cancer. 2016;61:146-56.

14. Usman Ali M, Miller J, Peirson L, et al. Screening for lung cancer: A systematic review and meta-analysis. Prev Med. 2016;89:301-14.

15. Riaz IB, Badgett RG. Epidural Corticosteroid Injections for Radiculopathy and Spinal Stenosis. Ann Intern Med. 2016;164:634-5.

16. Cochrane Library. Table 8.7.a: Possible approach for summary assessments of the risk of bias for each important outcome (across domains) within and across studies. 2011. (Accessed January 12, 2020, at http:// handbook-5-1.cochrane.org/chapter_8/table_8_7_a_possible_approach_ for_summary_assessments_of_the.htm)

17. Cornell JE, Mulrow CD, Localio R, et al. Random-effects meta-analysis of inconsistent effects: a time for change. Ann Intern Med. 2014; 160:26770 .

18. Cornell University. The OpenCPU System: Towards a Universal Interface for Scientific Computing through Separaration of Concerns. Cornell University; 2014. (Accessed January 12, 2020, at https://arxiv.org/ abs/1406.4806.)
19. Schwarzer G. meta: General Package for Meta-Analysis 4.9-9.: The Comprehensive R Archive Network; 2019.

20. Deeks JJ, Macaskill P, Irwig $\mathbf{L}$. The performance of tests of publication bias and other sample size effects in systematic reviews of diagnostic test accuracy was assessed. J Clin Epidemiol. 2005;58:882-93.

21. Higgins JP, Thompson SG, Deeks JJ, Altman DG. Measuring inconsistency in meta-analyses. BMJ. 2003;327:557-60.

22. Egger M, Davey Smith G, Schneider M, Minder C. Bias in metaanalysis detected by a simple, graphical test. BMJ. 1997;315:629-34.

23. Rucker G, Schwarzer G, Carpenter J. Arcsine test for publication bias in meta-analyses with binary outcomes. Stat Med. 2008;27:746-63.

24. Guyatt G, Oxman AD, Ak1 EA, et al. GRADE guidelines: 1. IntroductionGRADE evidence profiles and summary of findings tables. $J$ Clin Epidemiol. 2011;64:383-94.

25. Pinto RZ, Maher CG, Ferreira ML, et al. Epidural corticosteroid injections in the management of sciatica: a systematic review and metaanalysis. Ann Intern Med. 2012;157:865-77.

26. Doroudi M, Pinsky PF, Marcus PM. Lung Cancer Mortality in the Lung Screening Study Feasibility Trial. JNCI Cancer Spectr. 2018;2:pky042.

27. Infante M, Cavuto S, Lutman FR, et al. Long-Term Follow-up Results of the DANTE Trial, a Randomized Study of Lung Cancer Screening with Spiral Computed Tomography. Am J Respir Crit Care Med. 2015; 191:1166-75.

28. Wille MM, Dirksen A, Ashraf $\mathbf{H}$, et al. Results of the Randomized Danish Lung Cancer Screening Trial with Focus on High-Risk Profiling. Am J Respir Crit Care Med. 2016;193:542-51.

29. Pastorino U, Silva M, Sestini S, et al. Prolonged lung cancer screening reduced 10-year mortality in the MILD trial: new confirmation of lung cancer screening efficacy. Ann Oncol. 2019;30:1672.

30. Paci E, Puliti D, Lopes Pegna A, et al. Mortality, survival and incidence rates in the ITALUNG randomised lung cancer screening trial. Thorax. 2017;72:825-31.

31. Becker N, Motsch E, Trotter A, et al. Lung cancer mortality reduction by LDCT screening-Results from the randomized German LUSI trial. Int $J$ Cancer. 2019.

32. Yang $\mathbf{W}$, Gian $\mathbf{F}$, Teng $\mathbf{J}$, et al. Community-based lung cancer screening with low-dose CT in China: Results of the baseline screening. Lung Cancer. 2018;117:20-6.

33. Gohagan $\mathbf{J}$, Marcus $\mathbf{P}$, Fagerstrom R, et al. Baseline findings of a randomized feasibility trial of lung cancer screening with spiral CT scan vs chest radiograph: the Lung Screening Study of the National Cancer Institute. Chest. 2004;126:114-21.

34. Gohagan JK, Marcus PM, Fagerstrom RM, et al. Final results of the Lung Screening Study, a randomized feasibility study of spiral CT versus chest X-ray screening for lung cancer. Lung Cancer. 2005;47:9-15.

35. Infante M, Lutman FR, Cavuto S, et al. Lung cancer screening with spiral CT: baseline results of the randomized DANTE trial. Lung Cancer. 2008;59:355-63.

36. Infante M, Cavuto S, Lutman FR, et al. A randomized study of lung cancer screening with spiral computed tomography: three-year results from the DANTE trial. Am J Respir Crit Care Med. 2009; 180:445-53.

37. Infante M, Chiesa G, Solomon D, et al. Surgical procedures in the DANTE trial, a randomized study of lung cancer early detection with spiral computed tomography: comparative analysis in the screening and control arm. J Thorac Oncol. 2011;6:327-35.

38. National Lung Screening Trial Research Team, Aberle DR, Berg CD, et al. The National Lung Screening Trial: overview and study design. Radiology. 2011;258:243-53.

39. National Lung Screening Trial Research Team, Aberle DR, Adams AM, et al. Baseline characteristics of participants in the randomized national lung screening trial. J Natl Cancer Inst. 2010;102:1771-9.

40. Patz EF, Jr., Pinsky P, Gatsonis C, et al. Overdiagnosis in low-dose computed tomography screening for lung cancer. JAMA Intern Med. 2014; 174:269-74.

41. National Lung Screening Trial Research Team, Church TR, Black WC, et al. Results of initial low-dose computed tomographic screening for lung cancer. N Engl J Med. 2013;368:1980-91.

42. Pinsky PF, Bellinger CR, Miller DP, Jr. False-positive screens and lung cancer risk in the National Lung Screening Trial: Implications for shared decision-making. J Med Screen. 2018;25:110-2.

43. van Iersel CA, de Koning HJ, Draisma G, et al. Risk-based selection from the general population in a screening trial: selection criteria, recruitment and power for the Dutch-Belgian randomised lung cancer multi-slice CT screening trial (NELSON). Int J Cancer. 2007;120:868-74.

44. van de Wiel JC, Wang Y, Xu DM, et al. Neglectable benefit of searching for incidental findings in the Dutch-Belgian lung cancer screening trial 
(NELSON) using low-dose multidetector CT. Eur Radiol. 2007;17:147482 .

45. van Klaveren RJ, Oudkerk M, Prokop M, et al. Management of lung nodules detected by volume CT scanning. N Engl J Med. 2009;361:22219.

46. Horeweg N, Scholten ET, de Jong PA, et al. Detection of lung cancer through low-dose CT screening (NELSON): a prespecified analysis of screening test performance and interval cancers. Lancet Oncol. 2014;15:1342-50.

47. Xu DM, Gietema $\mathbf{H}$, de Koning $\mathbf{H}$, et al. Nodule management protocol of the NELSON randomised lung cancer screening trial. Lung Cancer. 2006;54:177-84.

48. Yousaf-Khan U, van der Aalst C, de Jong PA, et al. Final screening round of the NELSON lung cancer screening trial: the effect of a 2.5-year screening interval. Thorax. 2017;72:48-56.

49. Van't Westeinde SC, Horeweg N, De Leyn P, et al. Complications following lung surgery in the Dutch-Belgian randomized lung cancer screening trial. Eur J Cardiothorac Surg. 2012;42:420-9.

50. Pedersen JH, Ashraf H, Dirksen A, et al. The Danish randomized lung cancer CT screening trial-overall design and results of the prevalence round. J Thorac Oncol. 2009;4:608-14.

51. Saghir Z, Dirksen A, Ashraf H, et al. CT screening for lung cancer brings forward early disease. The randomised Danish Lung Cancer Screening Trial: status after five annual screening rounds with low-dose CT. Thorax. 2012;67:296-301.

52. Heleno B, Siersma V, Brodersen J. Estimation of Overdiagnosis of Lung Cancer in Low-Dose Computed Tomography Screening: A Secondary Analysis of the Danish Lung Cancer Screening Trial. JAMA Intern Med. 2018;178:1420-2.

53. Lopes Pegna A, Picozzi G, Mascalchi M, et al. Design, recruitment and baseline results of the ITALUNG trial for lung cancer screening with lowdose CT. Lung Cancer. 2009;64:34-40.

54. Lopes Pegna A, Picozzi G, Falaschi F, et al. Four-year results of lowdose CT screening and nodule management in the ITALUNG trial. J Thorac Oncol. 2013;8:866-75.

55. Pastorino U, Rossi M, Rosato V, et al. Annual or biennial CT screening versus observation in heavy smokers: 5-year results of the MILD trial. Eur J Cancer Prev. 2012;21:308-15.

56. Sverzellati N, Silva M, Calareso G, et al. Low-dose computed tomography for lung cancer screening: comparison of performance between annual and biennial screen. Eur Radiol. 2016;26:3821-9.

57. Pastorino U, Sverzellati N, Sestini S, et al. Ten-year results of the Multicentric Italian Lung Detection trial demonstrate the safety and efficacy of biennial lung cancer screening. Eur J Cancer. 2019;118:142-8.

58. Becker N, Motsch E, Gross ML, et al. Randomized study on early detection of lung cancer with MSCT in Germany: study design and results of the first screening round. J Cancer Res Clin Oncol. 2012;138: 1475-86.

59. Becker N, Motsch E, Gross ML, et al. Randomized Study on Early Detection of Lung Cancer with MSCT in Germany: Results of the First 3 Years of Follow-up After Randomization. J Thorac Oncol. 2015;10:890-6.

60. National Lung Screening Trial Research Team. Lung Cancer Incidence and Mortality with Extended Follow-up in the National Lung Screening Trial. J Thorac Oncol. 2019;14:1732-42.
61. Sedgwick P, Marston L. How to read a funnel plot in a meta-analysis. BMJ. 2015;351:h4718.

62. Howlader N, Noone AM, Krapcho M, et al. SEER Cancer Statistics Review, 1975-2016. Bethesda, MD: National Cancer Institute; 2016.

63. Schroder FH, Hugosson J, Roobol MJ, et al. Screening and prostate cancer mortality: results of the European Randomised Study of Screening for Prostate Cancer (ERSPC) at 13 years of follow-up. Lancet. 2014;384:2027-35.

64. Nelson HD, Fu R, Cantor A, Pappas M, Daeges M, Humphrey L. Effectiveness of Breast Cancer Screening: Systematic Review and Metaanalysis to Update the 2009 U.S. Preventive Services Task Force Recommendation. Ann Intern Med. 2016;164:244-55.

65. Fitzpatrick-Lewis D, Ali MU, Warren R, Kenny M, Sherifali D, Raina P. Screening for Colorectal Cancer: A Systematic Review and Meta-Analysis. Clin Colorectal Cancer. 2016;15:298-313.

66. Ten Haaf K, de Koning HJ. Overdiagnosis in lung cancer screening: why modelling is essential. J Epidemiol Community Health. 2015;69:1035-9.

67. American College of Radiology. Lung CT Screening Reporting \& Data System (Lung-RADS). (Accessed April 10, 2020, at https://www.acr.org/ Clinical-Resources/Reporting-and-Data-Systems/Lung-Rads.)

68. Pinsky PF, Gierada DS, Black W, et al. Performance of Lung-RADS in the National Lung Screening Trial: a retrospective assessment. Ann Intern Med. 2015;162:485-91.

69. Heijnsdijk EAM, Csanadi M, Gini A, et al. All-cause mortality versus cancer-specific mortality as outcome in cancer screening trials: A review and modeling study. Cancer Med. 2019;8:6127-38.

70. Pinsky PF, Church TR, Izmirlian G, Kramer BS. The National Lung Screening Trial: results stratified by demographics, smoking history, and lung cancer histology. Cancer. 2013;119:3976-83.

71. U.S. Preventive Services Task Force. Final Research Plan for Lung Cancer: Screening. 2018. (Accessed February 15, 2020, at https:// www.uspreventiveservicestaskforce.org/Page/Document/final-researchplan/lung-cancer-screening1)

72. Field JK, Duffy Sw, Baldwin DR, et al. The UK Lung Cancer Screening Trial: a pilot randomised controlled trial of low-dose computed tomography screening for the early detection of lung cancer. Health Technol Assess. 2016;20:1-146.

73. Robbins HA, Berg CD, Cheung LC, Chaturvedi AK, Katki HA. Identification of Candidates for Longer Lung Cancer Screening Intervals Following a Negative Low-Dose Computed Tomography Result. J Natl Cancer Inst. 2019;111:996-9.

74. Patz EF, Jr., Greco E, Gatsonis C, Pinsky P, Kramer BS, Aberle DR. Lung cancer incidence and mortality in National Lung Screening Trial participants who underwent low-dose CT prevalence screening: a retrospective cohort analysis of a randomised, multicentre, diagnostic screening trial. Lancet Oncol. 2016;17:590-9.

75. Tammemagi MC, Katki HA, Hocking WG, et al. Selection criteria for lung-cancer screening. N Engl J Med. 2013;368:728-36.

76. Barta JA, Powell CA, Wisnivesky JP. Global Epidemiology of Lung Cancer. Ann Glob Health. 2019;85.

77. Centers for Medicare \& Medicaid Services. MEDCAC Meeting 4/30/2014Lung Cancer Screening with Low Dose Computed Tomography. 2014. (Accessed February 2, 2020, at https://www.cms.gov/medicare-coverage-database/details/medcac-meeting-details.aspx?MEDCACId=68)

Publisher's Note: Springer Nature remains neutral with regard to jurisdictional claims in published maps and institutional affiliations. 